\title{
CONFORMALLY INVARIANT SYSTEMS OF NONLINEAR PDE OF LIOUVILLE TYPE
}

\author{
S. Chanillo and M.K.-H. Kiessling
}

\begin{abstract}
We establish a strict isoperimetric inequality and a Pohozaev-Rellich identity for the system

$$
-\Delta u_{\imath}(x)=\exp \left(\sum_{j \in \mathcal{I}} \gamma^{\imath, j} u_{\jmath}\right), \quad x \in \mathbb{R}^{2},
$$

$i \in \mathcal{I}=\{1, \ldots, N\}$, under certain reasonable conditions on the $\gamma^{\imath, 3}$ and $u_{\imath}$. Thus we prove that under these conditions, all solutions $u_{\imath}$ are radial symmetric and decreasing about some point.
\end{abstract}

\section{Introduction}

Systems of nonlinear elliptic PDEs of Liouville type (we shall call them "L-systems") are natural generalizations of Liouville's equation [Liou]

$$
-\Delta u(x)=e^{u}, \quad x \in \mathbb{R}^{2}
$$

for real-valued $u$. Incidentally, (1.1) is the simplest special case of an Lsystem. Many basic properties of (1.1) are reflected in any L-system as well. In particular, our results for L-systems - and their proofs - are readily explained by the model (1.1). Therefore, to begin with we devote some attention to (1.1).

We recall that (1.1) is invariant under translations, rotations and dilations in the Euclidean plane. With the possible exception of the origin (and $\infty),(1.1)$ is also invariant under Kelvin transforms. If one demands that the origin and $\infty$ are included, so that invariance under the full Euclidean conformal group prevails, this automatically implies that $\int \exp u d x=8 \pi$. Under this condition one can stereographically project (1.1) to $S^{2}$, and by various techniques, e.g. [On], [OsPhS], [CaLo], [Be], it can be proved that all solutions are given by a certain stereographical/conformal map of the constant function on $S^{2}$. This result is equivalent to a purely geometrical result similar to Obata's ([O]) for $S^{n}$. However, as pointed out by Chen and $\mathrm{Li}$ ([CheLi, Remark 1]), the results for $S^{2}$ do not extend to (1.1) without additional conditions. 
Interestingly, the additional condition that is needed is considerably milder than the demand of full Euclidean conformal invariance, or a growth condition for $u$ as $|x| \rightarrow \infty$. Chen and $\mathrm{Li}$ ([CheLi]) proved the following theorem.

THEOREM 1.1. Let $u \in L_{\text {loc }}^{1}\left(\mathbb{R}^{2}\right)$ be a solution of (1.1), satisfying the finite-mass condition

$$
\int_{\mathbb{R}^{2}} e^{u} d x<\infty
$$

Then $u$ is radial symmetric and decreasing about some point in $\mathbb{R}^{2}$.

This result is decisive for solving (1.1),(1.2) completely because it reduces the problem to a simple ODE problem with known solution. Thus, Chen and Li conclude, as a corollary of Theorem 1.1, that all solutions of (1.1),(1.2) are given by

$$
u\left(x \mid x_{0} ; \lambda\right)=-2 \ln \left(\left[1+\lambda^{2}\left|x-x_{0}\right|^{2}\right] / \lambda 2 \sqrt{2}\right),
$$

with $\lambda>0$ an arbitrary scale factor, and $x_{0} \in \mathbb{R}^{2}$ the arbitrary location of the maximum of $u$. It is readily checked that (1.3) is an orbit for the full Euclidean conformal group, and $\int \exp u d x=8 \pi$ for (1.3).

Clearly, Theorem 1.1 is also of interest in its own right. Chen and Li's proof of Theorem 1.1 is based on a variant of the moving plane method, which they adapted to certain peculiarities of the two-dimensionality of the problem. As such it relies heavily on maximum principles for elliptic PDE. For the moving plane method in other space dimensions and for rather general PDE problems, see Gidas, Ni, Nirenberg ([GNNi]) and C.-M. Li ([Li]). We mention also that Chou and Wan ([ChoW]) gave a direct proof that all solutions of (1.1),(1.2) are given by (1.3), which in turn implies Theorem 1.1 above. Their proof is tailored specifically for Liouville's equation. It uses Liouville's general solution ([Liou]) and is based entirely on complex analysis.

In section 2 of this paper we prove Theorem 1.1 in yet another way, i.e. without the use of the moving plane method, or any complex analysis. Instead we use an isoperimetric inequality in its strict form and show that any putative solution which is not radial and decreasing about any point would violate a Rellich-Pohozaev identity for (1.1),(1.2). Our proof is conceptually very simple, with subtleties coming in through technical aspects of the proof. Our technique has the advantage that it applies under certain conditions to L-systems as well, which is the main theme of our paper.

In the remainder of our paper, we consider the generic L-system

$$
-\Delta u_{i}(x)=\prod_{j \in \mathcal{I}} \exp \left(\gamma^{i, s} u_{j}\right), \quad x \in \mathbb{R}^{2},
$$


with $N$ finite mass conditions,

$$
\int_{\mathbb{R}^{2}} \exp \left(u_{i}\right) d x<\infty, \quad i \in \mathcal{I}
$$

Here, $\left\{\gamma^{i, j}\right\} \equiv \gamma \in G L_{N}(\mathbb{R})$, satisfying

$$
\sum_{j \in \mathcal{I}} \gamma^{i, j}=1, \quad i \in \mathcal{I}
$$

The $N$ conditions (1.6) define an affine subspace of $G L_{N}(\mathbb{R})$, which we denote by $\Gamma_{N}$. The matrix $\gamma$ may either be fixed or, more generally, depend on some parameter, $\lambda \in \mathbb{R}$, say. In the latter case, (1.4)-(1.6) is a nonlinear eigenvalue problem. We anticipate here that we are going to prove, in a similar way as we prove Theorem 1.1, that under certain conditions on the $\gamma$ an analog of Theorem 1.1 holds for the system (1.4)-(1.6). In the following we will explain why we believe that such a result is of interest.

Systems of equations (1.4), under slightly more general conditions which include (1.6) as a special case, find their applications in the physics of charged particle beams, e.g. [Ben], [KiL]. However, we find it interesting that, like Liouville's equation, they have an obvious geometrical significance. A solution $N$-tuple $\left\{u_{i}\right\}$ of (1.4), (1.6) defines a set of $N$ metrics, all of which are conformally equivalent to the standard one on $\mathbb{R}^{2}$. The metrics pertain to curvature functions $K_{i} \propto \exp \left(\sum_{j \neq i} \gamma^{i, j} u_{j}\right)$ which are not prescribed but are determined by the solutions $u_{j}$.

The system (1.4)-(1.6) poses some interesting mathematical problems. First of all, there always exists the solution family $u_{i}=u$, for all $i$, which implies that $u$ solves (1.1),(1.2), whence $u$ is given by (1.3). Clearly, we then recover full Euclidean conformal invariance. However, we also see that for this special solution family, (1.4), (1.5) are invariant under all changes of $\gamma$ satisfying (1.6), with fixed $u_{i}$. This leads to the obvious question whether the reverse is true.

Q.1: Can the solution family $u_{i}=u$, for all $i$, be characterized as being the only solution family of (1.4),(1.5) which is invariant under all changes of $\gamma \in \Gamma_{N}$ ?

This question is easily answered in the affirmative (see section 5). The answer is of some interest, however, because other solutions do exist. For instance, set $\gamma=\operatorname{id}_{N} \in \Gamma_{N}$, so that the $N$ equations (1.4) decouple into $N$ independent equations (1.1). Then the conditions (1.5) guarantee via Theorem 1.1 that each $u_{i}$ is given in the form (1.3), but this time typically $u_{i} \neq u_{j}$ for $i \neq j$. Thus we are led to ask: 
Q.2: For which $\gamma$ is $u_{i}=u$, for all $i$, the only solution family of (1.4)(1.6), and for which $\gamma$ is each $u_{i}$ given in the form (1.3) without implying $u_{i}=u$. for all $i$ ?

So far all solutions showed up with each $u_{i}$ being given in the form (1.3). For (1.1),(1.2) there are no other solutions, but for L-systems the situation is different. Consider indeed the special case $\gamma \in \Gamma_{2}$,

$$
\gamma=\left(\begin{array}{cc}
\gamma^{1,1} & \gamma^{1,2} \\
\gamma^{2,1} & \gamma^{2,2}
\end{array}\right)=\frac{1}{4}\left(\begin{array}{ll}
2+n+n^{2} & 2-n-n^{2} \\
2-n-n^{2} & 2+n+n^{2}
\end{array}\right)
$$

with $n \in \mathbb{N}$. The conformal orbit (1.3) at $u_{i}=\ln |J|^{2}+\ln 2=-2 \ln (1+$ $\left.|x|^{2}\right)+\ln 8, i=1,2$, provides us with a special solution to (1.4),(1.5),(1.7). Notice that $|J|^{2}$ is the Jacobian of the stereographic projection $S^{2} \rightarrow \mathbb{R}^{2}$. Projecting (1.4),(1.5),(1.7) from $\mathbb{R}^{2}$ to $S^{2}$ maps $\ln |J|^{2}+\ln 2$ into the unique constant solution for the projected equations. Linearizing the projected equations around the constant solution shows it can be continued iff for some $\alpha \in$ spec $(\gamma)$ the linear problem on $S^{2},-\Delta \phi=2 \alpha \phi$ has a nontrivial solution whose mean over $S^{2}$ vanishes. This requires $\operatorname{spec}(2 \gamma) \cap \operatorname{spec}_{S^{2}}^{\prime}(-\Delta) \neq \emptyset$; explicitly, we need $\alpha \in\{1,3,6, \ldots, k(k+1) / 2, \ldots\}$ for at least one $\alpha$. The eigenvalues of (1.7) are $\alpha_{1}=1$ and $\alpha_{2}=n(n+1) / 2$, whence two different continuations are possible. Now, 1 is an eigenvalue of all $\gamma \in \Gamma_{N}$, and the corresponding continuation of the constant solution in the direction of the first spherical harmonics gives just the conformal orbit (1.3). However, the eigenvalue $n(n+1) / 2$ of (1.7) opens the possibility to continue into a totally different solution branch which begins with an $n^{\text {th }}$ order spherical harmonic. The continuation of the $u_{i}$ into this branch may require a simultaneous nontrivial continuation of $\gamma$, resulting in a nonlinear eigenvalue problem. This example shows that Q.2 is an interesting problem.

Systems of the form (1.4)-(1.6) seem to have a richer solution structure than (1.1),(1.2). The solutions of $(1.1),(1.2)$ are classified completely. The most general question is thus:

Q.3: Can one classify all solutions for L-systems of the form (1.4)-(1.6)? If so. what are the solution classes?

We notice that for $(1.1),(1.2)$ the classification question was answered by proving that all solutions are radial symmetric and decreasing about one point, which reduced the problem to a solvable ODE. The possibility to classify all solutions in terms of an ODE does not seem to offer itself for (1.4)-(1.6) in view of the discussion around (1.7). Nevertheless, part of the solution manifold may be classified that way, and ODEs simplify the discussion significantly even though a completely solvable ODE is generally not to be expected. In any event, a natural question to ask is: 
Q.4: Under which conditions on $\gamma$ is each $u_{i}$ radial symmetric about some point $x_{0}^{i}$ ?

We may even ask:

Q.5: Under which conditions is each $u_{i}$ radial symmetric and decreasing about some point $x_{0}^{i}$ ?

Going one step further, we ask:

Q.6: Under which conditions is each $u_{i}$ radial symmetric and decreasing about the same point, i.e., $x_{0}^{i}=x_{0}$, for all $i$ ?

Recalling now the classification of solutions of (1.1),(1.2), we may ask

Q.7: Under the conditions which answer Q.5, is each $u_{i}$ given in the form (1.3)?

and similarly

Q.8: Under the conditions which answer $Q .6$, is $u_{i}=u$, for all $i$, the only solution family?

In sections 3-5. we are going to produce some partial answers to these questions. In section 3, we address Q.5 and Q.6. We will not be able to answer Q.5 and Q.6 in full, but by a generalization of our technique of proof of Theorem 1.1, we prove the following. We denote by $\Gamma_{N}^{+}$the convex closed subspace of $\Gamma_{N}$ which consists of matrices with non-negative entries, and by $\dot{\Gamma}_{N}^{+}$its interior.

THEOREM 1.2. Let $\gamma \in \Gamma_{N}^{+}$be symmetric, with all $\gamma^{i, i} \neq 0$. Then each $u_{i}$, with $i=1, \ldots, N$, is radial symmetric and decreasing about some point $x_{0}^{i}$. If in addition $\gamma \in \dot{\Gamma}_{N}^{+}$, then all $x_{0}^{i}$ coincide.

Interestingly, unlike for (1.1),(1.2), our results which lead to Theorem 1.2 do not imply that (1.4)-(1.6) is invariant under the full Euclidean conformal group, in general. This in turn implies that we were not able to answer Q.7 and Q.8 affirmatively, for otherwise full conformal invariance of (1.4)-(1.6) would prevail. However, we will be able to prove, in section 4, the following: THEOREM 1.3. Let $\gamma \in \Gamma_{N}^{+}$. Under the hypothesis that $(1.4)-(1.6)$ is invariant under the full Euclidean conformal group, we have

$$
\int_{\mathbb{R}^{2}} \prod_{j \in \mathcal{I}} \exp \left(\gamma^{i, j} u_{j}\right) d x=8 \pi, \quad i \in \mathcal{I} .
$$

If moreover $N=2, \gamma \in \Gamma_{N}^{+}, \gamma$ symmetric and $\gamma^{1,1}<\gamma^{1,2}$, then $u_{i}=u$, for $i=1,2$, with $u$ given by (1.3), is the only solution family to (1.4)-(1.6).

If $\left\{\gamma^{i, j}\right\}$ is not symmetric, or not even in $\Gamma_{N}^{+}$, it is unclear whether the technique to prove Theorems $1.2,1.3$ applies.

Finally, we can answer Q.1 in full. In section 5 we prove: 
THEOREM 1.4. For any $N$, there is only one family of solutions of (1.4)-(1.6) which is invariant under all changes of $\gamma \in \Gamma_{N}$. This family is given by $u_{i}=u$ for $i=1, \ldots, N$, with $u$ given by (1.3). Therefore, invariance under all changes of $\gamma \in \Gamma_{N}$ also implies full conformal invariance of (1.4)-(1.6).

\section{The Liouville Equation}

Turning first to the scalar case, we note that unlike [GNNi] or [Li] who imposed asymptotic conditions on $u$, [CheLi] made use of a lower a priori estimate on $\left\|e^{u}\right\|_{L^{1}}$ for solutions $u$ of (1.1), due to Ding ([CheLi]). This enabled them to obtain sufficient asymptotic control of $u$ to apply the moving plane method. Interestingly, Ding's estimate, which is based on a weak form of an isoperimetric inequality, brings geometrical and functional analytic ideas into the proof. A strengthening of Ding's inequality is readily done, giving the strict inequality $\left\|e^{u}\right\|_{L^{1}}>8 \pi$ for any solution $u$ of (1.1),(1.2) which is not radial decreasing about any point in $\mathbb{R}^{2}$. On the other hand, assuming now a certain reasonable asymptotic behavior of $u$ and $\nabla u$ at large $|x|$ allows one to apply a Rellich-Pohozaev identity, see $[\mathrm{R}]$, [Po], giving the identity $\left\|e^{u}\right\|_{L^{1}}=8 \pi$ for any solution of (1.1),(1.2). Thus one obtains a short proof of radial symmetry and decrease about some point in $\mathbb{R}^{2}$ of any solution of (1.1),(1.2); however, only under certain asymptotic conditions on $u$. We remark that in a finite disk $B_{R} \subset \mathbb{R}^{2}$ a proof of this kind has been given by Keady and Bandle [B, p. 203].

Now, the interesting question is whether one can drop asymptotic conditions at infinity and yet conclude the proof along the same lines, i.e. a strict Ding inequality vs. Rellich-Pohozaev identity. Chen and Li have shown how to obtain the asymptotic behavior posed as conditions in [Li] and [GNNi], but their estimates are not sufficient to establish a Pohozaev-Rellich identity. In the remainder of this section. we obtain the relevant asymptotic control directly from (1.1),(1.2).

Our assumptions are rather weak. We require $u \in L_{l o c}^{1}\left(\mathbb{R}^{2}\right)$ together with (1.2), so that (1.1) has a meaning at least in the sense of distributions. We begin by recalling a result of Brezis and Merle ([BrMe, Thm. 2]), and Cor. 3.3 in [ChLi]:

Lemma 2.1. Let $u \in L_{\text {loc }}^{1}\left(\mathbb{R}^{2}\right)$ be a solution of (1.1). Assume (1.2) holds. Then $u^{+} \in L^{\infty}\left(\mathbb{R}^{2}\right)$.

Various regularity results follow immediately from Theorem 2 of [BrMe] and standard elliptic regularity as discussed in, e.g. [GiT]. Since $u^{+} \in L^{\infty}$, also $\Delta u \in L^{\infty}$, whence $u \in C_{l o c}^{1, \alpha}$. It even follows from here that $u \in C^{\infty}$ by bootstrapping. Notice, however, that Theorem 2 of [BrMe], i.e. Lemma 2.1, 
does not imply any bound on $|u|$. The regularity allows one to arrive at the next lemma, which is due to Ding ([CheLi]). Its proof is based on a weak form of an isoperimetric inequality. A variant for finite domains is found in $[\mathrm{B}]$.

Lemma 2.2. Let $u$ be any solution of (1.1), satisfying (1.2). Then,

$$
\int_{\mathbb{R}^{2}} e^{u} d x \geq 8 \pi
$$

Lemma 2.2 can be sharpened in the following way.

LEMMA 2.3. If $u$ is a solution of (1.1),(1.2) which is not radial symmetric and decreasing about any point, then the strict inequality

$$
\int_{\mathbb{R}^{2}} e^{u} d x>8 \pi
$$

holds.

Proof: First, we observe that a solution $u$ of (1.1),(1.2) which is not radial symmetric and decreasing cannot be radial symmetric at all. Indeed, assume $u$ is radial symmetric about some point $x_{0}$ but not everywhere decreasing away from $x_{0}$. By translation invariance of $(1.1),(1.2)$ we can choose $x_{0}$ to be the origin. Let $B_{R}$ be the ball with radius $R$, centered at $x_{0}$. Then, by (1.1), Green's theorem, and using $u \in C_{\text {loc }}^{1, \alpha}$, we have for any finite $R$,

$$
0<\int_{B_{R}} e^{u} d x=-\int_{B_{R}} \Delta u d x=-2 \pi R u^{\prime}(R)
$$

in contradiction to the assumption that $u$ is somewhere not decreasing. Hence, by the assumptions made in Lemma 2.3 we can now even assume that $u$ is not radial symmetric at all.

Let $u^{*}$ denote the equi-measurable, radial symmetric non-increasing rearrangement of $u$, centered at $x=0$. Let $\Lambda_{c}=\{x \mid u(x) \geq c\}, \Lambda_{c}^{*}=\{x \mid$ $u^{*}(x) \geq c$, with $R_{c}$ the radius of $\Lambda_{c}^{*}$. Having the regularity of $u$ noted below Lemma 2.1, we conclude $\partial \Lambda_{c} \in C^{1, \alpha}$ except for a set of values of $c$ of measure 0 , which is guaranteed by Sard's theorem. Thus the unit outward normal $\nu(x)$ to $\partial \Lambda_{c}$ exists at almost all $\partial \Lambda_{c}$. Furthermore, by (1.2), the $\Lambda_{c}$ are of finite measure.

Then, since $u$ is not radial about any point, for almost every $c$ we have, by the strict isoperimetric inequality, Green's theorem, and (1.1),

$$
-\left.2 \pi R_{c} u^{* \prime}\right|_{R_{c}}=-\int_{\partial \Lambda_{c}^{*}} \partial_{r} u^{*} d \sigma<-\int_{\partial \Lambda_{c}}\langle\nu, \nabla u\rangle d \sigma=-\int_{\Lambda_{c}} \Delta u d x=\int_{\Lambda_{c}} e^{u} d x .
$$

In (2.4) and in the following, $\langle a, b\rangle$ denotes the standard scalar product in $\mathbb{R}^{2}$. Now set 


$$
M(r)=\int_{B_{r}(0)} e^{u^{*}} d x
$$

We see with

$$
\int_{\Lambda_{c}^{*}} e^{u^{*}} d x=\int_{\Lambda_{c}} e^{u} d x
$$

that (2.4) implies for almost every $r$,

$$
2 \pi r u^{* \prime}(r)>-M(r) .
$$

Arguing now as in [CheLi, Lemma (1.1)], but using (2.7) instead, we easily get

$$
2 \pi r M^{\prime \prime}(r)>2 \pi M^{\prime}(r)-M(r) M^{\prime}(r)
$$

for almost all $r>0$. We now integrate (2.8) from $r=0$ to $r=\infty$. Using the fact that $e^{u^{*}} \in L^{1}\left(\mathbb{R}^{2}\right)$ is radial decreasing, we see that $\lim _{r \rightarrow \infty} r M^{\prime}(r)=0$. This gives

$$
\int_{\mathbb{R}^{2}} e^{u^{*}} d x>8 \pi
$$

With (2.6) this proves Lemma 2.3.

We now turn to controlling the asymptotic behavior of solutions $u$ of (1.1),(1.2) and their gradients. We notice that Lemma 2.1 combined with a standard argument of harmonic analysis implies the following representation lemma.

LEMma 2.4. Let $u$ be a solution of (1.1). Assume (1.2) holds. Then $u$ satisfies

$$
u(x)=-\frac{1}{2 \pi} \int_{\mathbb{R}^{2}}(\ln |x-y|-\ln |y|) e^{u(y)} d y+C
$$

for some constant $C$.

This representation lemma is proved in [CheLi, proof of Lemma 1.2].

The asymptotic behavior of $u$ now obtains as a corollary of Lemma 2.4, see [CheLi, Lemma 1.2]; it appears in a more general form as Lemma 1 in [ChKi], in which form we will need it in later sections.

Corollary 2.1. It follows from (2.10) and (1.2) that

$$
\lim _{|x| \rightarrow \infty} \frac{u(x)}{\ln |x|}=-\frac{1}{2 \pi} \int_{\mathbb{R}^{2}} e^{u} d x
$$

uniformly as $|x| \rightarrow \infty$.

This leads to

LEMma 2.5. Let $u$ be a solution of (1.1), (1.2). Then there exists a $\varepsilon(u)>0$, and for any $\varepsilon^{\prime}>0$ there exists a $r_{0}\left(\varepsilon^{\prime} ; u\right)>0$ and a constant $C\left(\varepsilon^{\prime} ; u\right)$ such that for $|x|>r_{0}$. 


$$
\begin{aligned}
e^{u(x)} & \leq C|x|^{-4+\varepsilon^{\prime}} \\
& \leq C|x|^{-2-\varepsilon} .
\end{aligned}
$$

Proof: By Corollary 2.1 and Lemma 2.2, for any $\varepsilon^{\prime}>0$ we have

$$
\frac{u(x)}{\ln |x|} \leq-4+\varepsilon^{\prime},
$$

uniformly as $|x| \rightarrow \infty$. This proves (2.12a), whence (2.12b) follows.

Remark 1: The estimate (2.12b) for $\exp u$, being here a trivial consequence of (2.12a), follows alternatively by Lemma 2.4 and (1.2) alone. We have included it for the following main reason. In the ensuing proofs of gradient estimates we do not need (2.12a), but only $\exp (u) \leq C|x|^{-2-\varepsilon}$ asymptotically, for some fixed $\varepsilon$ and $C$. This is decisive for extending our results to L-systems, for which only an estimate of the form (2.12b), but not (2.12a), will be available.

Remark 2: If $u$ is not radial decreasing about any point, then there exists a $\delta(u)>0$, a $r_{0}(u)>0$ and a constant $C(u)$ such that the sharper bound

$$
e^{u(x)} \leq C(u)|x|^{-4-\delta} \quad \text { for } \quad|x|>r_{0}
$$

holds. Indeed, let $u$ not be radial decreasing about any point. Then by Lemma 2.3 and Corollary 2.1 there exists a $\delta(u)>0$ such that

$$
\frac{u(x)}{\ln |x|} \leq-4-\delta,
$$

uniformly as $|x| \rightarrow \infty$. Our claim (2.14) follows.

We proceed with the derivation of gradient estimates.

Lemma 2.6. For $u$ a solution to (1.1),(1.2),

$$
\limsup _{|x| \rightarrow \infty}|x||\nabla u| \leq \frac{1}{2 \pi} \int_{\mathbb{R}^{2}} e^{u} d x
$$

Proof: We get from the representation of $u$, Lemma 2.4,

$$
|\nabla u(x)| \leq \frac{1}{2 \pi} \int_{\mathbb{R}^{2}} \frac{e^{u}}{|x-y|} d y .
$$

Multiplying (2.17) by $|x|$, and a simple rewriting of the r.h.s. gives

$$
|x||\nabla u(x)| \leq \frac{1}{2 \pi} \int_{\mathbb{R}^{2}} e^{u} d x+\frac{1}{2 \pi} \int_{\mathbb{R}^{u}}\left(\frac{|x|}{|x-y|}-1\right) e^{u} d y .
$$

By the triangle inequality, the second integral in (2.18) is bounded in absolute value by

$$
\frac{1}{2 \pi} \int_{\mathbb{R}^{2}} \frac{|y|}{|x-y|} e^{u} d y .
$$

We now show that 


$$
\lim _{|x| \rightarrow \infty} \frac{1}{2 \pi} \int_{\mathbb{R}^{2}} \frac{|y|}{|x-y|} e^{u} d y=0
$$

from which the lemma follows.

We split the domain of integration in (2.19) as follows: $\mathbb{R}^{2}=\Omega_{1} \cup \Omega_{2} \cup \Omega_{3}$, with $\Omega_{1}=\{y|| y|<| x \mid / 2\}, \Omega_{2}=\{y|| x|/ 2 \leq| y|\leq 2| x \mid\}$, and $\Omega_{3}=\{y \mid$ $|y|>2|x|\}$. We estimate the contribution from $\Omega_{1}$ by using $\exp u(y) \leq$ $C|y|^{-2-\varepsilon}$, see $(2.12 \mathrm{~b})$, with $0<\epsilon<1$,

$$
\int_{\Omega_{1}} \frac{|y|}{|x-y|} e^{u} d y \leq \frac{C}{|x|} \int_{\Omega_{1}}|y| e^{u} d y \leq \frac{C^{\prime}}{|x|} \int_{0}^{|x|}|y|^{-\varepsilon} d(|y|) \leq C^{\prime \prime}|x|^{-\varepsilon} .
$$

Thus, as $|x| \rightarrow \infty$, r.h.s.(2.21) vanishes, and so must l.h.s.(2.21). The contribution from $\Omega_{3}$ is dominated by

$$
\int_{\Omega_{3}} \frac{|y|}{|x-y|} e^{u} d y \leq C \int_{|y|>2|x|} e^{u} d y
$$

which vanishes as $|x| \rightarrow \infty$, by hypothesis (1.2). The contribution from $\Omega_{2}$ is estimated by using $\exp u(y) \leq C|y|^{-2-\varepsilon}$, see $(2.12 \mathrm{~b})$, again, and is bounded by,

$$
\int_{\Omega_{2}} \frac{|y|}{|x-y|} e^{u} d y \leq \frac{C}{|x|^{1+\varepsilon}} \int_{|y|<4|x|} \frac{d y}{|y|} \leq C|x|^{-\varepsilon}
$$

for some $\varepsilon<1$ if $|x|$ is large enough. Clearly r.h.s.(2.23) $\rightarrow 0$ as $|x| \rightarrow \infty$. This concludes the proof of Lemma 2.6.

LEMMA 2.7. For a solution $u$, we have

$$
\lim _{|x| \rightarrow \infty}\langle x, \nabla u\rangle=-\frac{1}{2 \pi} \int_{\mathbb{R}^{2}} e^{u} d x
$$

uniformly in $x$.

Proof: Let $\theta=x /|x|, \tau=y /|y|,|x|=|y|$. We first show that for $|x|>R$ and $|x-y|<|x| / 10$, we have,

$$
|x||\nabla u(x)-\nabla u(y)| \leq C|\theta-\tau|+C^{\prime}|x|^{-\varepsilon} .
$$

The proof essentially follows that of Lemma 2.6.

By the representation for $u$, Lemma 2.4,

$$
\nabla u(x)=-\frac{1}{2 \pi} \int_{\mathbb{R}^{2}} \frac{x-z}{|x-z|^{2}} e^{u(z)} d z .
$$

Thus,

$$
|\nabla u(x)-\nabla u(y)| \leq \frac{1}{2 \pi} \int_{\mathbb{R}^{2}} e^{u(z)}\left|\frac{x-z}{|x-z|^{2}}-\frac{y-z}{|y-z|^{2}}\right| d z .
$$


We break up the domain of integration in the above integral exactly as in the proof of Lemma 2.6. (Notice the integration variable is now z.) For the integration over $\Omega_{1}$ we apply the mean value theorem to the difference in the integrand. Remembering that $|x-y|<|x| / 10$, we see easily that the integral over $\Omega_{1}$ is dominated by,

$$
\int_{\mathbb{R}^{2}} e^{u(z)} \frac{|x-y|}{|x|^{2}} d z \leq C \frac{|x-y|}{|x|^{2}} .
$$

The integral over $\Omega_{2}$ is dominated by

$$
\int_{|z| \sim|x|} e^{u(z)}\left(\frac{1}{|x-z|}+\frac{1}{|y-z|}\right) d z \leq \frac{C}{|x|^{1+\varepsilon}} .
$$

The final estimate above was identical to that made in the proof of Lemma 2.6. Use was made of $e^{u(z)} \leq C|x|^{-2-\varepsilon}$ on $\Omega_{2}$, which follows from $(2.12 \mathrm{~b})$. For the contribution from $\Omega_{3}$ we make use of the mean value theorem on the difference in the integrand and see easily that the integral is dominated by,

$$
C|x-y| \int_{|z|>2|x|} \frac{e^{u(z)}}{|z|^{2}} d z \leq C^{\prime} \frac{|x-y|}{|x|^{2}} \int_{\mathbb{R}^{2}} e^{u} d x \leq C^{\prime \prime} \frac{|x-y|}{|x|^{2}},
$$

the last step follows by (1.2).

Now fix $\theta \in S^{1}$. By Corollary 2.1,

$$
\lim _{t \rightarrow \infty} \frac{u(t \theta)}{\ln t}=-\frac{1}{2 \pi} \int_{\mathbb{R}^{2}} e^{u} d x
$$

Thus by L'Hopital's Rule,

$$
\lim _{t \rightarrow \infty} t \frac{d}{d t} u(t \theta)=\lim _{|x| \rightarrow \infty}\langle x, \nabla u\rangle=-\frac{1}{2 \pi} \int_{\mathbb{R}^{2}} e^{u} d x
$$

for $x=|x| \theta$.

We now establish uniformity of the above limit. We show that there exist $R$ and $\delta$ such that, if $|x|>R$ and $|\theta-\tau|<\delta$, then

$$
|\langle x, \nabla u(x)\rangle-\langle y, \nabla u(y)\rangle|<\varepsilon .
$$

Indeed, by our earlier estimate,

$$
\begin{aligned}
|\langle x, \nabla u(x)\rangle-\langle y, \nabla u(y)\rangle| & \leq|x||\theta-\tau||\nabla u(x)|+|y||\nabla u(x)-\nabla u(y)| \\
& \leq|x||\nabla u(x)||\theta-\tau|+|\theta-\tau|+C|x|^{-\varepsilon} .
\end{aligned}
$$

By Lemma 2.6, the last expression above is at most $C^{\prime} \delta+C|x|^{-\varepsilon}$. Thus our claim (2.32) follows for suitably large $R$ and small $\delta$. Since $S^{1}$ is compact, uniformity of the limit in Lemma 2.7 now follows.

Lemmata 2.6 and 2.7 imply 
COROLlary 2.2. Uniformly in $x$ we have,

$$
\lim _{|x| \rightarrow \infty}|x||\nabla u|=\frac{1}{2 \pi} \int_{\mathbb{R}^{2}} e^{u} d x .
$$

Proof: Using Lemma 2.7, uniformly in $x$ we have

$$
\lim _{|x| \rightarrow \infty}|\langle x, \nabla u\rangle|=\frac{1}{2 \pi} \int_{\mathbb{R}^{2}} e^{u} d x .
$$

Furthermore

$$
\frac{1}{2 \pi} \int_{\mathbb{R}^{2}} e^{u} d x=\lim _{|x| \rightarrow \infty}|\langle x, \nabla u\rangle| \leq \liminf _{|x| \rightarrow \infty}|x||\nabla u| \leq \limsup _{|x| \rightarrow \infty}|x||\nabla u| \leq \frac{1}{2 \pi} \int_{\mathbb{R}^{2}} e^{u} d x .
$$

The last inequality in (2.37) is Lemma 2.6. Our Corollary 2.2 follows.

Let $\Lambda_{c}=\{x: u(x) \geq c\}$, where $c \ll-1$. By Corollary 2.1 it follows that if $x \in \partial \Lambda_{c}$, then $|x| \geq R(c)$ with $R(c)$ large. For such $x$, it then follows from Corollary 2.2 that $\nabla u \neq 0$. By $u \in C_{l o c}^{1, \alpha}$ we easily see that therefore $\partial \Lambda_{c} \in C^{1, \alpha}$. Thus the unit outward normal $\nu(x)$ to $\partial \Lambda_{c}$ exists at all $x \in \partial \Lambda_{c}$ for $c$ sufficiently negative.

LEMMA 2.8. Let $\nu(x)$ be the unit outward normal to $\partial \Lambda_{c}$ at $x$, and let $\theta=x /|x|$. We have

$$
\lim _{c \rightarrow-\infty}\langle\theta, \nu\rangle=1
$$

uniformly in $x$.

Remark: Lemma 2.8 implies that asymptotically the $\partial \Lambda_{c}$ become confocal circles.

Proof: On $\partial \Lambda_{c}$, we have $\nabla u=-\nu|\nabla u|$. Thus,

$$
|\langle x, \nabla u\rangle|=|x||\nabla u||\langle\theta, \nu\rangle| \text {. }
$$

Therefore

$$
\lim _{|x| \rightarrow \infty}|\langle\theta, \nu\rangle|=\lim _{|x| \rightarrow \infty} \frac{|\langle x, \nabla u\rangle|}{|x||\nabla u|} .
$$

By Lemma 2.7 and Corollary 2.2, r.h.s. $(2.40) \rightarrow 1$. Note further that, by Lemma 2.7 , by $\nabla u=-\nu|\nabla u|$ and by continuity of $u$, we have $\langle\theta, \nu\rangle \geq 0$ on $\partial \Lambda_{c}$. Thus we can drop the absolute bars on l.h.s.(2.40), and we have our lemma.

We are now in a position to prove the second main lemma, which is a limit of a finite-volume Rellich--Pohozaev identity.

LEMMA 2.9. Let $u$ be any solution to (1.1), satisfying (1.2). Then,

$$
\int_{\mathbb{R}^{2}} e^{u} d x=8 \pi
$$


Proof: For $u$ a solution of (1.1),(1.2), we have the partial differential identity

$$
\operatorname{div}(\langle x, \nabla u\rangle \nabla u)-|\nabla u|^{2}-\frac{1}{2}\left\langle x, \nabla|\nabla u|^{2}\right\rangle=-\left\langle x, \nabla\left(e^{u}\right)\right\rangle .
$$

We integrate this identity over $\Lambda_{c}$. By Green's theorem we arrive at the finite-volume Rellich-Pohozaev identity, which in our case reads,

$$
\frac{1}{2} \int_{\partial \Lambda_{c}}\langle x, \nu\rangle|\nabla u|^{2} d \sigma_{c}+\int_{\partial \Lambda_{c}}\langle x, \nu\rangle e^{u} d \sigma_{c}=2 \int_{\Lambda_{c}} e^{u} d x
$$

where $d \sigma_{c}$ is arc length measure on $\partial \Lambda_{c}$.

By Lemma 2.8, for $|x|$ large enough the domain $\Lambda_{c}$ is star-shaped w.r.t the origin. Thus we may represent $\partial \Lambda_{c}$ in polar coordinates by $r=f(\varphi)$, with $u(f(\varphi), \varphi)=c$. Furthermore, $d \sigma_{c} / d \varphi=\left(r^{2}+f^{\prime}(\varphi)^{2}\right)^{1 / 2}$. An elementary computation shows $\left(f^{\prime}(\varphi)\right)^{2}=\left(u_{\varphi} / u_{r}\right)^{2}$. Thus,

$$
\frac{1}{r} \frac{d \sigma_{c}}{d \varphi}=\frac{1}{\left|u_{r}\right|}\left(u_{r}^{2}+\left(\frac{1}{r} u_{\varphi}\right)^{2}\right)^{1 / 2}=\frac{|\nabla u|}{\left|u_{r}\right|}=\frac{|x||\nabla u|}{|\langle x, \nabla u\rangle|} .
$$

It follows from Corollary 2.2 that

$$
\lim _{c \rightarrow-\infty} \frac{1}{r} \frac{d \sigma_{c}}{d \varphi}=1
$$

Now the second integral on the left in (2.43) can be written as

$$
\int_{0}^{2 \pi}\langle\theta, \nu\rangle e^{u}|x| \frac{d \sigma_{c}}{d \varphi} d \varphi
$$

But $|\langle\theta, \nu\rangle| \leq 1$, and by Lemma 2.5, (2.12b), $e^{u} \leq C|x|^{-2-\varepsilon}$. Thus the integrand above is $O\left(|x|^{-\varepsilon}\right)$, as $|x| \rightarrow \infty$. Thus by the dominated convergence theorem,

$$
\lim _{c \rightarrow-\infty} \int_{\partial \Lambda_{c}}\langle x, \nu\rangle e^{u} d x=0 .
$$

Since $e^{u} \in L^{1}$, it follows that

$$
\lim _{c \rightarrow-\infty} \int_{\Lambda_{c}} e^{u} d x=\int_{\mathbb{R}^{2}} e^{u} d x
$$

Thus as $c \rightarrow-\infty$, the right side of $(2.43)$ is $2 \int_{\mathbb{R}^{2}} e^{u} d x$. Now the first integral on the left side of $(2.43)$ can be written as

$$
\frac{1}{2} \int_{0}^{2 \pi}\langle\theta, \nu\rangle|x|^{2}|\nabla u|^{2} \frac{1}{|x|} \frac{d \sigma_{c}}{d \varphi} d \varphi
$$

Applying Corollary 2.2, Lemma 2.8, and the identity (2.44), we have by the dominated convergence theorem,

$$
\lim _{c \rightarrow-\infty} \int_{\partial \Lambda_{c}}\langle x, \nu\rangle|\nabla u|^{2} d \sigma_{c}=\left(\frac{1}{2 \pi} \int_{\mathbb{R}^{2}} e^{u} d x\right)^{2} \int_{0}^{2 \pi} d \varphi .
$$

Thus taking limits in (2.43) as $c \rightarrow-\infty$ we have 


$$
\pi\left(\frac{1}{2 \pi} \int_{\mathbb{R}^{2}} e^{u} d x\right)^{2}=2 \int_{\mathbb{R}^{2}} e^{u} d x
$$

Since $\int_{\mathbb{R}^{2}} e^{u} d x<\infty$, Lemma 2.9 is proved.

We now have all ingredients to prove Theorem 1.1.

Proof of Theorem 1.1: Assume $u$ is a solution of (1.1),(1.2) which is not radial and decreasing about any point. We arrive at a contradiction. Indeed, by Lemma 2.3, our assumption on $u$ implies $\int_{\mathbb{R}^{2}} e^{u} d x>8 \pi$ strictly. But this contradicts Lemma 2.9 by which all solutions to $(1.1),(1.2)$ obey the identity $\int_{\mathbb{R}^{2}} e^{u} d x=8 \pi$. Therefore, any solution is radial symmetric and decreasing about a point in $\mathbb{R}^{2}$. The proof of Theorem 1.1 is complete.

\section{Radial Symmetry for Generic L-systems}

We now turn to $(1.4)-(1.6)$. We assume $u_{i} \in L_{\text {loc }}^{1}\left(\mathbb{R}^{2}\right), i \in \mathcal{I}=\{1, \ldots, N\}$, so that the $u_{i}$ solve (1.4) (1.6) at least in the sense of distributions. Our goal in this section is to prove that for a reasonable class of $\gamma$ all solutions of (1.4)-(1.6) are radial symmetric and decreasing about the same point. Our proof follows the pattern given in the previous section for the scalar Liouville equation. The generalization is, however, not completely straightforward, as there are some interesting twists.

We first show that under certain conditions on $\gamma$, Theorem 2 of Brezis and Merle [BrMe] applies to each $u_{i}$. This is the analog of Lemma 2.1.

LEMMA 3.1. Let $u_{i} \in L_{\text {loo }}^{1}\left(\mathbb{R}^{2}\right), i \in \mathcal{I}$, solve $(1.4)-(1.6)$, and let $\gamma \in \Gamma_{N}^{+}$, with diagonal elements $\gamma^{i, i} \in(0,1]$. Then $u_{i}^{+} \in L^{\infty}\left(\mathbb{R}^{2}\right)$.

Proof: We first observe that if $\gamma^{i, i}=1$ for some $i$, then the $i^{\text {th }}$ equation (1.4) and the $i^{\text {th }}$ equation (1.5) decouple from the remainder, reducing to (1.1) and (1.2). for which Lemma 2.1 verifies our claim. Let thus $\gamma^{i, i} \in(0,1)$. By (1.5). we then find

$$
\exp \left(\gamma^{i, i} u_{i}\right) \in L^{p^{\prime}}\left(\mathbb{R}^{2}\right)
$$

with $p^{\prime}=1 / \gamma^{i, i}$. Now define for $j \in \mathcal{I} \backslash\{i\}$ the new matrix elements

$$
\tilde{\gamma}^{i, j}=\frac{\gamma^{i, j}}{1-\gamma^{i, i}} \in(0, \infty) \text {. }
$$

Then for each $i \in \mathcal{I}$,

$$
\sum_{j \in \mathcal{I} \backslash\{i\}} \tilde{\gamma}^{i, j}=1 .
$$

Thus by Hölder's inequality and (1.5), for each $i \in \mathcal{I}$, 


$$
\int_{\mathbb{R}^{2}} \prod_{j \in \mathcal{I} \backslash\{i\}} \exp \left(\widetilde{\gamma}^{i, j} u_{j}(x)\right) d x \leq \prod_{j \in \mathcal{I} \backslash\{i\}}\left[\int_{\mathbb{R}^{2}} \exp \left(u_{j}(x)\right) d x\right]^{\widetilde{\gamma}^{i, j}}<\infty
$$

whence

$$
\prod_{j \in \mathcal{I} \backslash\{i\}} \exp \left(\gamma^{i, j} u_{j}(x)\right) \in \mathrm{L}^{p}\left(\mathbb{R}^{2}\right)
$$

with $p=1 /\left(1-\gamma^{i, i}\right)$ being the conjugate exponent to $p^{\prime}$.

Next, we define

$$
U_{i}=\gamma^{i, i} u_{i}
$$

which is nontrivial since $\gamma^{i, i} \neq 0$. We also define

$$
V_{i}=\gamma^{i, i} \prod_{j \in \mathcal{I} \backslash\{i\}} \exp \left(\gamma^{i, j} u_{j}(x)\right)
$$

Therefore, by (3.6) and (3.7), for each $i \in \mathcal{I}$ equation (1.4) can be rewritten in the form

$$
-\Delta U_{i}=V_{i}(x) e^{U_{2}}
$$

with $e^{U_{\imath}} \in L^{p^{\prime}}$ and $V_{i} \in L^{p}$, see (3.1) and (3.5). Also, $U_{i} \in L_{l o c}^{1}\left(\mathbb{R}^{2}\right)$. Therefore Theorem 2 of $[\mathrm{BrMe}]$ applies to each $U_{i}$, whence

$$
U_{i}^{+} \in L^{\infty}\left(\mathbb{R}^{2}\right) \text {. }
$$

This completes the proof.

By the same observation as presented after Lemma 2.1, we now conclude that all $u_{i} \in C_{\text {loc }}^{1, \alpha}$, thus in $C^{\infty}$.

We now prepare for the analog of Lemmata 2.2 and 2.3. We define $M_{i}$ and $m_{i}$ via

$$
8 \pi m_{i}=M_{i}=\int_{\mathbb{R}^{2}} \prod_{j \in \mathcal{I}} \exp \left(\gamma^{i, j} u_{j}(x)\right) d x .
$$

Notice that by Hölder inequality and (1.5) we have

$$
M_{i} \leq \prod_{j \in \mathcal{I}}\left[\int_{\mathbb{R}^{2}} \exp \left(u_{j}(x)\right) d x\right]^{\gamma^{2, j}}<\infty .
$$

Lemma 3.2. Under the hypothesis stated in Lemma 3.1, and the additional hypothesis that $\gamma^{i, j}=\gamma^{j, i}$, each solution vector $\left\{u_{i}\right\}$ of (1.4)-(1.6) satisfies the inequality

$$
\sum_{(i, j) \in \mathcal{I} \times \mathcal{I}} m_{i} \gamma^{i, j} m_{j}-\sum_{i \in \mathcal{I}} m_{i} \geq 0
$$

Under the additional hypothesis that at least one of the $u_{i}$ is not radial symmetric and decreasing about some point, the strict inequality 


$$
\sum_{(i, j) \in \mathcal{I} \times \mathcal{I}} m_{i} \gamma^{i, j} m_{j}-\sum_{i \in \mathcal{I}} m_{i}>0
$$

holds.

Proof: We define the new functions

$$
u_{i}=\sum_{j \in \mathcal{I}} \gamma^{i, j} u_{j}
$$

which by (1.4) satisfy

$$
-\Delta w_{i}=\sum_{j \in \mathcal{I}} \gamma^{i, j} \exp \left(w_{j}\right)
$$

We apply the isoperimetric inequality to the $w_{i}$. Let thus $\Lambda_{c}^{i}=\left\{x \mid w_{i} \geq\right.$ $\left.c_{i}\right\}$. By (3.11), $\int_{\Lambda_{c}^{2}} d x<\infty$. Let $w_{i}^{*}$ denote the equi-measurable, radial symmetric non-increasing rearrangement of $w_{i}$, centered at the origin, and let $\Lambda_{c}^{i^{*}}=\left\{x \mid w_{i}^{*} \geq c_{i}\right\}$, a ball of radius $R_{c}^{i}$. By the regularity of the $w_{i}$, the outward normal $\nu$ to $\partial \Lambda_{c}^{i}$ exists with the exception of $c$ values in at most a set of measure zero. Then, by the isoperimetric inequality, Green's theorem, (3.15), and again the isoperimetric inequality (noting $\gamma \in \Gamma_{N}^{+}$), we have

$$
\begin{aligned}
-\left.2 \pi R_{c}^{i} w_{i}^{* \prime}\right|_{R_{c}^{2}} & =-\int_{\partial \Lambda_{c}^{z^{*}}} \partial_{r^{2} w_{i}^{*} d \sigma} \\
& \leq-\int_{\partial \Lambda_{c}^{i}}\left\langle\nu, \nabla w_{i}\right\rangle d \sigma \\
& =-\int_{\Lambda_{c}^{\imath}} \Delta w_{i} d x \\
& =\sum_{j \in \mathcal{I}} \gamma^{i, j} \int_{\Lambda_{c}^{2}} \exp \left(w_{j}\right) d x \\
& \leq \sum_{j \in \mathcal{I}} \gamma^{i, j} \int_{\Lambda_{c}^{2 *}} \exp \left(w_{j}^{*}\right) d x
\end{aligned}
$$

Now we set

$$
M_{i}(r)=\int_{B_{r}(0)} \exp \left(w_{i}^{*}\right) d x
$$

We have from $(3.16)$,

$$
2 \pi r w_{i}^{* \prime}(r) \geq-\sum_{j \in \mathcal{I}} \gamma^{i, j} M_{j}(r)
$$


which we use to estimate

$$
2 \pi r M_{i}^{\prime \prime}(r) \geq 2 \pi M_{i}^{\prime}(r)-M_{i}^{\prime}(r) \sum_{j \in \mathcal{I}} \gamma^{i, j} M_{j}(r)
$$

for all $r>0$. We now sum (3.19) w.r.t. $i \in \mathcal{I}$. Using the symmetry of $\gamma$, we find

$$
\sum_{(i, j) \in \mathcal{I} \times \mathcal{I}} M_{i}^{\prime}(r) \gamma^{i, j} M_{j}(r)=\frac{1}{2} \sum_{(i, j) \in \mathcal{I} \times \mathcal{I}}\left(M_{i}(r) \gamma^{i, j} M_{j}(r)\right)^{\prime} .
$$

Now we integrate $\sum_{i}(3.19)$ from $r=0$ to $r=\infty$. Using the fact that $\exp \left(w_{i}^{*}\right) \in L^{1}\left(\mathbb{R}^{2}\right)$ is radial decreasing, we see that $\lim _{r \rightarrow \infty} r M_{i}^{\prime}(r)=0$. This gives, with (3.20) and with

$$
\int_{\mathbb{R}^{2}} \exp \left(w_{i}^{*}\right) d x=\int_{\mathbb{R}^{2}} \exp \left(w_{i}\right) d x=M_{i}
$$

the inequality

$$
\sum_{(i, j) \in \mathcal{I} \times \mathcal{I}} M_{i} \gamma^{i, j} M_{j}-8 \pi \sum_{i \in \mathcal{I}} M_{i} \geq 0
$$

With the definition of the $m_{i}$ in (3.10), this proves (3.12).

To prove (3.13), we notice that if at least one of the $u_{i}$ is not radial symmetric and decreasing about some point, it is not radial symmetric at all. This follows again by Gauss' theorem and the positivity of the r.h.s. of (1.4), cf. the proof of Lemma 2.3. We may thus assume that at least for one $i=i_{0}$, the $u_{i_{0}}$ is not radial symmetric at all. In that case, for this $i_{0},(3.16)$ becomes a strict inequality for almost every $c$. Hence, (3.22) then becomes a strict inequality. This proves (3.13), whence Lemma 3.2 in full.

We will next establish a Pohozaev-Rellich identity for (1.4)-(1.6) which says that all solutions satisfy (3.12) for the special case of identity in the right-hand side. It turns out to be here of advantage to work with the equation (1.4) rather than (3.15). For this we need to control the asymptotic behavior of the gradients of the $u_{i}$.

Lemma 3.3. Let $u_{i} \in L_{\text {loc }}^{1}\left(\mathbb{R}^{2}\right)$, solve (1.4)-(1.6), and let $\gamma \in \Gamma_{N}^{+}$, with diagonal elements $\gamma^{i, i} \in(0,1]$. Then each $u_{i}$ satisfies

$$
u_{i}(x)=-\frac{1}{2 \pi} \int_{\mathbb{R}^{2}}(\ln |x-y|-\ln |y|) \prod_{j \in \mathcal{I}} \exp \left(\gamma^{i, j} u_{j}(y)\right) d y+C_{i}
$$

for some constant $C_{i}$.

Proof: We rewrite (1.4) into the form (3.8). We notice that by (1.5), the r.h.s. of (3.8) is in $L^{1}$. By the proof of Lemma 3.1, $V_{i} \in L^{\infty}$, with $V_{i}$ defined 
in (3.7). The problem (3.8) with $V_{i} \in L^{\infty}$ and r.h.s. (3.8) $\in L^{1}$ is treated in [ChKi]. The rest of the proof is therefore identical to the corresponding part in the proof of Lemma 1 in [ChKi].

COROLLARY 3.1. Under the conditions of Lemma 3.1, for the solutions $u_{i}$, $i \in \mathcal{I}$ of (1.4)-(1.6), it follows from Lemma 3.1 that

$$
\lim _{|x| \rightarrow \infty} \frac{u_{i}(x)}{\ln |x|}=-\frac{M_{i}}{2 \pi}
$$

uniformly as $|x| \rightarrow \infty$.

Proof: Same proof as for Lemma 1 in [ChKi].

We obtain asymptotic control over the r.h.s. of (1.4) by (3.24).

LemMa 3.4. Let $\left\{u_{i}\right\}$ be a solution vector of (1.4)-(1.6), and let the hypotheses of Lemma 3.1 hold. Then there exist $\varepsilon_{i}>0$ and $r_{0}\left(w_{i}\right)>0$ and a constant $C_{i}$ such that for each $i$,

$$
\exp \left(w_{i}(x)\right) \leq C_{i}|x|^{-2-\varepsilon_{i}} \quad \text { for } \quad|x|>r_{0} .
$$

Proof: By (3.11) the $M_{i}$ exist. Using the representation Lemma 3.3 in the definition (3.10) of each $M_{i}$, with Corollary 3.1 we then see that the integrability, as $|x| \rightarrow \infty$, requires

$$
\frac{w_{i}(x)}{\ln |x|} \leq-2-\varepsilon_{i}
$$

uniformly as $|x| \rightarrow \infty$. Our Lemma 3.4 follows.

We proceed with gradient estimates.

Lemma 3.5. Under the hypotheses expressed in Lemma 3.1, for the solutions $u_{i}$ of (1.4)-(1.6) we have

$$
\lim _{|x| \rightarrow \infty}\left\langle x, \nabla u_{i}\right\rangle=-\frac{M_{i}}{2 \pi} .
$$

uniformly in $x$.

Proof: With minor modifications the same proof as for Lemma 2.7 applies. We only need to recall that the estimates go through with the weak decay rate $\exp \left(w_{i}\right) \sim|x|^{-2-\varepsilon}$, stated in Lemma 3.4.

LEMMA 3.6. Under the hypotheses expressed in Lemma 3.1, for the solutions $u_{i}$ of (1.4)-(1.6) we have

$$
\lim _{|x|-\infty}|x|\left|\nabla u_{i}\right|=\frac{M_{i}}{2 \pi}
$$

uniformly in $x$. 
Proof: The proof of Lemma 2.6 and Corollary 2.2 applies for the same reasons as given in the proof of Lemma 3.5.

The main lemma follows now.

LEMMA 3.7. Under the hypotheses expressed in Lemma 3.2, any solution vector $\left\{u_{i}\right\}$ of $(1.4)-(1.6)$ satisfies

$$
\sum_{(i, j) \in \mathcal{I} \times \mathcal{I}} m_{i} \gamma^{i, j} m_{j}-\sum_{i \in \mathcal{I}} m_{i}=0 .
$$

The proof of this lemma does not proceed exactly as the counterpart for Lemma 2.9. The reasons are: Since we have $N$ functions $u_{i}$, it is preferable not to single out any of them and to operate on $\Lambda_{c}^{i}$, as in the proof of Lemma 3.2. Rather we use disks. Second, the scheme of partial integrations is now applied directly to the $u_{i}$, not to the $w_{i}$ as in the proof of Lemma 3.2. Clearly, for Liouville's equation there is only one $u$, which is therefore identical to the corresponding $w$; whence the distinction made here is redundant and unimportant. It is vital for L-systems, though.

Proof of Lemma 3.7: For $\left\{u_{i}\right\}$ a solution of (1.4)-(1.6), we have the partial differential identity

$$
\operatorname{div}\left(\left\langle x . \nabla u_{j}\right\rangle \nabla u_{i}\right)=\left\langle\nabla u_{i},(1+\langle x, \nabla\rangle) \nabla u_{j}\right\rangle-\left\langle x, \nabla u_{j}\right\rangle \exp \left(\sum_{k \in \mathcal{I}} \gamma^{i, k} u_{k}\right) .
$$

We multiply (3.30) by $\gamma^{i, j}$, sum over $i$ and $j$. integrate over $B_{R}$, then, after some partial integrations, take the limit $R \rightarrow \infty$.

Green's theorem gives for the integral over the l.h.s. of (3.30)

$$
\int_{B_{R}} \operatorname{div}\left(\left\langle x, \nabla u_{j}\right\rangle \nabla u_{i}\right) d x=\int_{\partial B_{R}}|x|^{-1}\left\langle x, \nabla u_{j}\right\rangle\left\langle x, \nabla u_{i}\right\rangle d \sigma .
$$

Taking the limit $R \rightarrow \infty$. using Lemma 3.5 we get

$$
\lim _{R \rightarrow \infty} \text { r.h.s. }(3.31)=\frac{1}{2 \pi} M_{i} M_{j}
$$

and therefore

$$
\lim _{R \rightarrow \infty}\left\{\sum_{(i, j) \in \mathcal{I} \times \mathcal{I}} \gamma^{i, j} \text { r.h.s.(3.31) }\right\}=\frac{1}{2 \pi} \sum_{(i, j) \in \mathcal{I} \times \mathcal{I}} M_{i} \gamma^{i, j} M_{j} .
$$

The r.h.s. of $\sum_{(i, j) \in \mathcal{I} \times \mathcal{I}} \gamma^{i, j}(3.30)$ can be integrated entirely in terms of the $M_{i}$ as follows. We have for the last term, 


$$
\begin{aligned}
\sum_{(i, j) \in \mathcal{I} \times \mathcal{I}} \gamma^{i, j} \int_{B_{R}}\left\langle x, \nabla u_{j}\right\rangle \exp \left(\sum_{k \in \mathcal{I}} \gamma^{i, k} u_{k}\right) d x= \\
=\sum_{i \in \mathcal{I}} \int_{B_{R}}\left\langle x, \nabla \exp \left(\sum_{k \in \mathcal{I}} \gamma^{i, k} u_{k}\right)\right\rangle d x= \\
=\sum_{i \in \mathcal{I}} \int_{\partial B_{R}}\langle x, \nu\rangle \exp \left(\sum_{k \in \mathcal{I}} \gamma^{i, k} u_{k}\right) d \sigma \\
-2 \sum_{i \in \mathcal{I}} \int_{B_{R}} \exp \left(\sum_{k \in \mathcal{I}} \gamma^{i, k} u_{k}\right) d x
\end{aligned}
$$

Taking the limit $R \rightarrow \infty$ in (3.34) gives

$$
\lim _{R \rightarrow \infty} \text { r.h.s. }(3.34)=-2 \sum_{i \in \mathcal{I}} M_{i}
$$

The surface integral gives no contribution because of Lemma 3.4.

Finally, we now use the symmetry of $\gamma$. a partial integration and (1.4) to get

$$
\begin{aligned}
& \sum_{(i, j) \in \mathcal{I} \times \mathcal{I}} \gamma^{i, j} \int_{B_{R}}\left\langle\nabla u_{i},(1+\langle x, \nabla\rangle) \nabla u_{j}\right\rangle d x \\
& =\frac{1}{2} \sum_{(i, j) \in \mathcal{I} \times \mathcal{I}} \gamma^{i, j} \int_{\partial B_{R}}\langle x, \nu\rangle\left\langle\nabla u_{i}, \nabla u_{j}\right\rangle d \sigma \\
& =\frac{1}{2} \sum_{(i, j) \in \mathcal{I} \times \mathcal{I}} \gamma^{i, j} \int_{\partial B_{R}}\langle x, \nu\rangle\left\langle x, \nabla u_{i}\right\rangle\left\langle x, \nabla u_{j}\right\rangle|x|^{-2} d \sigma \\
& \quad+\frac{1}{2} \sum_{(i, j) \in \mathcal{I} \times \mathcal{I}} \gamma^{i, j} \int_{\partial B_{R}}\langle x, \nu\rangle\left\langle\nabla_{t a n} u_{i}, \nabla_{t a n} u_{j}\right\rangle d \sigma
\end{aligned}
$$

where $\nabla_{\text {tan }}$ denotes tangential derivative. Now Lemmata 3.5 and 3.6 tell us that

$$
|x|^{2}\left|\nabla_{\tan } u_{i}\right|^{2}=|x|^{2}\left|\nabla u_{i}\right|^{2}-\left\langle x, \nabla u_{i}\right\rangle^{2} \rightarrow 0
$$

uniformly as $|x| \rightarrow \infty$. Thus as $R \rightarrow \infty$,

$$
\int_{\partial B_{R}}\langle x, \nu\rangle\left\langle\nabla_{t a n} u_{i}, \nabla_{l a n} u_{j}\right\rangle d \sigma \rightarrow 0
$$

and therefore

$$
\lim _{R \rightarrow \infty} \text { 1.h.s. }(3.36)=\frac{1}{4 \pi} \sum_{(\imath, j) \in \mathcal{I} \times \mathcal{I}} M_{i} \gamma^{i, j} M_{j} .
$$

In total we get a Rellich-Pohozaev identity in the form 


$$
\sum_{(i, j) \in \mathcal{I} \times \mathcal{I}} M_{i} \gamma^{i, j} M_{j}-8 \pi \sum_{i \in \mathcal{I}} M_{i}=0
$$

With (3.10) this proves Lemma 3.7.

The proof of Theorem 1.2 is now obvious.

Proof of Theorem 1.2: By (3.13) of Lemma 3.2, and by Lemma 3.7, equation (3.29), the solutions $u_{i}$ of (1.4)-(1.6) have to be radial symmetric and non-increasing. As we have not excluded that some $\gamma^{i, i}=1$, some equations in (1.4)-(1.6) may decouple from the rest, so the centers of symmetry of the various $u_{i}$ need not coincide.

Now assume $\gamma \in \dot{\Gamma}_{N}^{+}$. Then $\gamma$ is irreducible. The r.h.s. of (1.4) then implies that the centers of radial symmetry must coincide. The proof is complete.

\section{Full Conformal Invariance and a Convexity Result}

Interestingly, the Rellich-Pohozaev identity (3.40) does not guarantee that (1.4)-(1.6) are fully conformal invariant, in contrast to the situation for (1.1),(1.2). On the other hand, demanding invariance of (1.4)-(1.6) under the full Euclidean conformal group, i.e. including Kelvin transforms, implies for $\gamma \in \Gamma_{N}$ the following result.

Lemma 4.1. Let $\gamma \in \Gamma_{N}^{+}$and demand full conformal invariance of (1.4)(1.6). Then

$$
M_{i}=8 \pi
$$

for all $i$.

Proof: We need to check invariance under Kelvin transforms

$$
x \rightarrow y=\frac{x}{|x|^{2}}
$$

together with

$$
w_{i}(x)=\sum_{j \in \mathcal{I}} \gamma^{i, j} u_{j}(x) \rightarrow-4 \ln |y|+\sum_{j \in \mathcal{I}} \gamma^{i, j} u_{j}\left(y /|y|^{2}\right) \equiv \tilde{w}_{i}(y) .
$$

Demanding the $\tilde{w}_{i}(y)$ be smooth at the origin, with (3.24) we get,

$$
\sum_{j \in \mathcal{I}} \gamma^{i, j} M_{j}=8 \pi
$$

for all $i$, and since $\gamma \in \Gamma_{N} \subset G L_{N}(\mathbb{R})$. we find (4.1), for all $i$. The proof is complete. 
We do not know at present whether (4.1) is true automatically under much weaker conditions than full conformal invariance. We also do not know whether Lemma 4.1 implies that $u_{i}=u$ for general $N$. However, for $N=2$ and under an ordering condition, we can conclude that $u_{i}=u$ for $i=1,2$. Thus we prove Theorem 1.3.

Proof of Theorem 1.3: Part 1 of Theorem 1.3 is proved by Lemma 4.1. The rest of the proof utilizes a convexity argument. We subtract (1.4) for $u_{2}$ from (1.4) for $u_{1}$, then multiply by $u_{1}-u_{2}$, and integrate over $\mathbb{R}^{2}$. Thus we obtain

$\int_{\mathbb{R}^{2}}\left|\nabla\left(u_{1}-u_{2}\right)\right|^{2} d x=\int_{\mathbb{R}^{2}}\left(u_{1}-u_{2}\right) e^{\gamma^{3,1} u_{1}+\gamma^{2,2} u_{2}}\left(e^{\left[\gamma^{1,2}-\gamma^{2,2}\right] u_{2}}-e^{\left[\gamma^{2,1}-\gamma^{1,1}\right] u_{1}}\right) d x$.

Now clearly, since by assumption $\gamma$ is symmetric and $\gamma^{1,1}<\gamma^{1,2}$, the r.h.s. of (4.5) is non-positive. Thus $u_{1}=u_{2}$, and the proof is complete.

\section{A Uniqueness Result}

We consider here question Q.1 and answer it in the affirmative. We notice that any $\gamma \in \Gamma_{N}$ can be written as

$$
\gamma=\mathrm{id}_{N}+g(\gamma)
$$

with $g \in M_{N}(\mathbb{R})$ satisfying

$$
\sum_{j \in \mathcal{I}} g^{i, j}=0
$$

for all $i \in \mathcal{I}$. Therefore also

$$
\gamma_{t}=\mathrm{id}_{N}+t g(\gamma) \in \Gamma_{N}
$$

with $t \in \mathbb{R}$. We notice further that 0 is an eigenvalue of $g$ with the constant vector as right eigenvector.

Proof of Theorem 1.4: Let $\left\{u_{i}\right\}$ be a solution $N$-tuple of (1.4)-(1.6) and assume that $\left\{u_{i}\right\}$ remains a solution under all changes $\gamma \rightarrow \gamma^{\prime} \in \Gamma_{N}$. Then, in particular, $\left\{u_{i}\right\}$ is invariant along rays of the form (5.3) with $g$ chosen such that 0 is a simple eigenvalue. Now we differentiate (1.4) w.r.t. the parameter $t$ and obtain

$$
0=\sum_{j=1}^{N} g^{i, j} u_{j}(x)
$$

Since, (5.2), 0 is a simple eigenvalue, with right eigenvector being the constant vector, we find that $u_{i}(x) \equiv u_{j}(x)$ for all $i, j$ is the only solution. This completes the proof of Theorem 1.4. 
Note added. After submission of this paper, P. Laurence and F. Pacella kindly pointed out to us the paper by P.-L. Lions ([Lio]), where the KeadyBandle argument is extended to more general nonlinearities $f$ for $-\Delta u(x)=$ $f(u), x \in B_{R} \subset \mathbb{R}^{2}$ and 0-Dirichlet b.c. The method has been extended to equations involving the $p$-Laplacian in $B_{R} \subset \mathbb{R}^{N}$, for $p=N$, in [KP].

Acknowledgement. The work of S.C. was supported through NSF Grant No. DMS 9401782, and that of M.K. through a DFG fellowship and through AFOSR Grant No. 92-J-0115. M.K. is indebted to J.L. Lebowitz for inviting him to spend Summer 1994 at Rutgers University and for his warm hospitality.

\section{References}

[B] C. BandLE, Isoperimetric Inequalities and Applications, Pitman, Boston, 1980.

[Be] W. BECKNER, to appear.

[Ben] W.H. BENNETT, Magnetically self-focussing streams, Phys. Rev. 45 (1934), $890-897$.

[BrMe] H. Brezis, F. Merle, Uniform estimates and blow-up behavior of solutions of $-\Delta u=V(x) e^{u}$ in two dimensions, Commun. PDE 16 (1991), 1223-1253.

[ClMP] E. Caglioti, P.-L. Lions, C. Marchioro, M. Pulvirenti, A special class of stationary flows for two-dimensional Euler equations: A statistical mechanical description, Commun. Math. Phys. 143 (1992), 501-525.

[CaLo] E.A. Carlen, M. Loss, Competing symmetries, the logarithmic HLS inequality, and Onofri's inequality on $S^{n}$, Geom. Funct. Anal. 2 (1992), 90-104.

[ChKi] S. Chanillo, M. Kiessling, Rotational symmetry of solutions of some nonlinear problems in statistical mechanics and in geometry, Commun. Math. Phys. 160:2 (1994), 217-238.

[ChLi] S. Chanillo, Y.Y. LI, Continuity of solutions of uniformly elliptic equations in $\mathbb{R}^{2}$, Manuscr. Math. 77 (1992), 415-433.

[CheLi] W. ChEn, C. LI, Classification of solutions of some nonlinear elliptic equations, Duke Math. J. 63 (1991), 615-622.

[ChoW] K.S. ChOU, T.Y.H. WAN, Asymptotic radial symmetry for solutions of $\Delta u+$ $e^{u}=0$ in a punctured disc, Pac. J. Math. 163 (1994), 269-276.

[ESp] G. EYINK, H. SPOHN, Negative temperature states and large-scale long-lived vortices in two-dimensional turbulence, J. Stat. Phys. 70 (1993), 833-886.

[GNNi] B. Gidas, W.M. NI, L. Nirenberg, Symmetry and related properties via the maximum principle, Commun. Math. Phys. 68 (1979), 209-243.

[GiT] D. Gilbarg, N.S. Trudinger, Elliptic Partial Differential Equations of Second Order, Springer Verlag, New York (1983).

[KP] C. KESAVAN, F. PaCELla, Symmetry of positive solutions of a quasilinear elliptic equation via isoperimetric inequalities, Appl. Anal. 54 (1994), 2737.

[Ki] M.K.-H. Kiessuing, Statistical mechanics of classical particles with logarithmic interactions, Commun. Pure Appl. Math. 46 (1993), 27-56.

[KiL] M.K.-H. KIessling, J.L. Lebowitz, Dissipative Stationary Plasmas: Kinetic Modeling, Bennett's Pinch, and generalizations, Phys. Plasmas 1 (1994), 1841-1849. 
[Li] C.-M. LI, Monotonicity and symmetry of solutions of fully nonlinear elliptic

equations on unbounded domains, Commun. PDE 16 (1991), 585-615.

[Lio] P.-L. Lions, Two geometrical properties of solutions of semilinear problems, Appl. Anal. 12 (1981), 267-272.

[Liou] J. LiouviLLe, Sur l'equation aux différences partielles $\partial^{2} \log \lambda / \partial u \partial v \pm \lambda / 2 a^{2}$ $=0$, J. Math. $18(1853), 71-72$.

[O] M. OBATA, The conjectures on conformal transformations of Riemannian manifolds, J. Diff. Geom. 6 (1971), 247-258.

[On] E. ONOFRI, On the positivity of the effective action in a theory of random surfaces, Commun. Math. Phys. 86 (1982), 321-326.

[OsPhS] B. Osgood, R. Phillips, P. SarnaK, Extremals of determinants of Laplacians, J. Funct. Anal. 80 (1988), 148-211.

[Po] S.I. Pohozaev, Eigenfunctions of the equation $\Delta u+\lambda f(u)=0$, Sov. Math. Dokl. 5 (1965), 1408-1411.

[R] F. Rellich, Darstellung der Eigenwerte von $\Delta u+\lambda u=0$ durch ein Randwertintegral, Math. Z. 46 (1940), 635-636.

Sagun Chanillo

Department of Mathematics

Rutgers University

New Brunswick, NJ 08903

USA
Michael K.-H. Kiessling

Department of Mathematics

Rutgers University

New Brunswick, NJ 08903

USA 